\title{
Front Matter: Volume 11065
}

, "Front Matter: Volume 11065," Proc. SPIE 11065, Saratov Fall Meeting 2018: Optical and Nano-Technologies for Biology and Medicine, 1106501 (3 June 2019); doi: $10.1117 / 12.2532343$

SPIE Event: International Symposium on Optics and Biophotonics VI: Saratov Fall SPIE. Meeting 2018, 2018, Saratov, Russian Federation 


\title{
PROGRESS IN BIOMEDICAL OPTICS AND IMAGING \\ Vol. 20 No. 55
}

\section{Saratov Fall Meeting 2018}

\section{Optical and Nano-Technologies for Biology and Medicine}

\author{
Elina A. Genina \\ Valery V. Tuchin
}

Editors

\section{4-28 September 2018 \\ Saratov, Russian Federation}

\section{Sponsored by}

Russian Foundation for Basic Research (Russian Federation) - Russian Academy of Sciences (Russian Federation) • SPIE • OSA-The Optical Society •IEEE - The Photonics Society • Russian Technology Platform "The Medicine of the Future" (Russian Federation) • Russian Technology Platform "Photonics" (Russian Federation) • European Technology Platform "Photonics21" - EPIC - European Photonics Industry Consortium • LLC SPE "Nanostructured Glass Technology" (Russian Federation)• RME "INJECT" LLC (Russian Federation) • LLC SPE Nanostructured Glass Technology (Russian Federation) • Technolnfo, Ltd. (Russian Federation) • BioVitrum, Ltd. (Russian Federation) • LLC SPE OESSP (Russian Federation)

Published by

SPIE

\section{Volume 11065}


The papers in this volume were part of the technical conference cited on the cover and title page. Papers were selected and subject to review by the editors and conference program committee. Some conference presentations may not be available for publication. Additional papers and presentation recordings may be available online in the SPIE Digital Library at SPIEDigitallibrary.org.

The papers reflect the work and thoughts of the authors and are published herein as submitted. The publisher is not responsible for the validity of the information or for any outcomes resulting from reliance thereon.

Please use the following format to cite material from these proceedings:

Author(s), "Title of Paper," in Saratov Fall Meeting 2018: Optical and Nano-Technologies for Biology and Medicine, edited by Elina A. Genina, Valery V. Tuchin, Proceedings of SPIE Vol. 11065 (SPIE, Bellingham, WA, 2019) Seven-digit Article CID Number.

ISSN: 1605-7422

ISSN: $2410-9045$ (electronic)

ISBN: 9781510628205

ISBN: 9781510628212 (electronic)

Published by

SPIE

P.O. Box 10, Bellingham, Washington 98227-0010 USA

Telephone +1360 676 3290 (Pacific Time) · Fax +1 3606471445

SPIE.org

Copyright @ 2019, Society of Photo-Optical Instrumentation Engineers.

Copying of material in this book for internal or personal use, or for the internal or personal use of specific clients, beyond the fair use provisions granted by the U.S. Copyright Law is authorized by SPIE subject to payment of copying fees. The Transactional Reporting Service base fee for this volume is $\$ 18.00$ per article (or portion thereof), which should be paid directly to the Copyright Clearance Center (CCC), 222 Rosewood Drive, Danvers, MA 01923. Payment may also be made electronically through CCC Online at copyright.com. Other copying for republication, resale, advertising or promotion, or any form of systematic or multiple reproduction of any material in this book is prohibited except with permission in writing from the publisher. The CCC fee code is $0277-$ $786 \times / 19 / \$ 18.00$.

Printed in the United States of America by Curran Associates, Inc., under license from SPIE.

Publication of record for individual papers is online in the SPIE Digital Library.

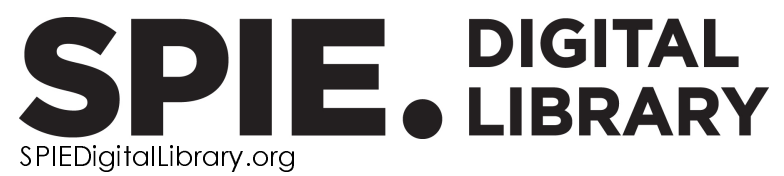

Paper Numbering: Proceedings of SPIE follow an e-First publication model. A unique citation identifier (CID) number is assigned to each article at the time of publication. Utilization of CIDs allows articles to be fully citable as soon as they are published online, and connects the same identifier to all online and print versions of the publication. SPIE uses a seven-digit CID article numbering system structured as follows:

- The first five digits correspond to the SPIE volume number.

- The last two digits indicate publication order within the volume using a Base 36 numbering system employing both numerals and letters. These two-number sets start with $00,01,02,03,04$, 05, 06, 07, 08, 09, OA, OB ... 0Z, followed by 10-1Z, 20-2Z, etc. The CID Number appears on each page of the manuscript. 


\section{Contents}

$\begin{aligned} \text { ix } & \text { Authors } \\ \text { xiii } & \text { Conference Committee } \\ \text { xvii } & \text { Introduction } \\ \text { xix } & \text { Organizers }\end{aligned}$

INVITED PAPERS

1106502 OCT lymphangiography based on speckle statistics evaluation (Invited Paper) [1 1065-61]

1106503 OCT-based three-dimensional strain mapping for elastography and relaxography (Invited Paper) [1 1065-62]

$1106504 \quad 5-A L A / P p I X$ fluorescent diagnostics of stressed-induced small and large intestine neoplasia in laboratorial rats in vivo (Invited Paper) [1 1065-91]

1106505 Research and development of effective optical technologies for diagnostics in dermatology (Invited Paper) [1 1065-102]

\section{BEST STUDENT POSTER AWARD WINNERS}

1106506 Optical coherence elastography as a new method for estimation of chemotherapy efficacy on triple-negative breast cancer in the experiment (Best Student Poster Award) [1 1065-15]

$1106507 \quad$ Effect of different macromolecules on viscous and microrheologic properties of blood at various temperatures (Best Student Poster Award) [1 1065-87]

1106508 Optical diagnostics of bile duct tissues state with tumor compression (Best Student Poster Award) [11065-33]

1106509 Effect of ethanol on the transport of methylene blue through the rat skin ex vivo (Best Student Poster Award) [1 1065-92]

OPTICAL TECHNOLOGIES IN DIAGNOSTICS

11065 OA Multispectral and autofluorescence RGB imaging for skin cancer diagnostics [1 1065-1] 
11065 OB Photodynamic diagnostics for stomach cancer detection in stress modeled lesions in vivo [11065-3]

11065 OC Phase delay measurements based on acousto-optic filtration of interference image in nearcommon-path interferometer [1 1065-8]

11065 OD Comparison of elastic properties of tissue samples in various pathological states using optical coherence elastography [11065-14]

11065 OE Blood coagulation estimation using the method of laser-speckle correlation [1 1065-2]

11065 OF Amplification of output signal of laser scanning speckle-microscope using gold nanoparticles for detection of Chlamydia trachomatis bacteria. Part 2: experiments [1 1065-27]

11065 0G Study of statistical properties of GB-speckles, generated on nucleotide sequences of omp1 gene of Chlamydia trachomatis, simulated using different algorithms of re-coding. Part I. Statistics of intensity fluctuations [1 1065-28]

$11065 \mathrm{OH} \quad$ Study of statistical properties of GB-speckles, generated on nucleotide sequences of omp1 gene of Chlamydia trachomatis, simulated using different algorithms of re-coding. Part II. Statistics of phase fluctuations [1 1065-29]

$1106501 \quad$ Application of s-LASCA technique for processing of GB-speckles, coding sequences of nucleotide sequences of ompl gene of Chlamydia trachomatis [1 1065-30]

$110650 \mathrm{~J} \quad$ Investigation of statistical characteristics of GB-speckles, coding nucleotide sequences of the gene GPCR of Lumpy Skin Disease virus [1 1065-31]

11065 OK Investigation of structural and statistical characteristics of interfering GB-speckles, coding the nucleotide sequences of the gene GPCR of Lumpy Skin Disease Virus [1 1065-38]

$11065 \mathrm{OL} \quad$ In-vivo pulse wave diagnostics of chicken embryo using high-frequency modulation of intensity of illuminating laser light [1 1065-39]

11065 OM Detection of viability of chicken embryo by method of laser Doppler diagnostics [1 1065-40]

11065 ON Detection of blood microcirculation of chicken embryo using LASCA-imaging with application of the new techniques of optical clearing [11065-41]

$1106500 \quad$ Amplification of output signal of laser scanning speckle-microscope using gold nanoparticles for detection of Chlamydia trachomatis bacteria. Part 1: theoretical study [1 1065-42]

11065 OP Study of statistical properties of GB-speckles coding the nucleotide sequences of the target genes of Avian Influenza Virus [1 1065-47]

$110650 Q \quad$ Laser speckle contrast imaging of abdominal organs in mouse model [1 1065-51]

11065 OR Fluorescence spectroscopy approach for blood influence compensation [1 1065-35]

11065 OS Pilot studies of age-related changes in blood perfusion in two different types of skin [1 1065-37] 
11065 OT Investigation of blood microcirculation parameters in patients with rheumatic diseases by videocapillaroscopy and laser Doppler flowmetry during cold pressor test [1 1065-52]

11065 OU Comparative in-vivo and in-vitro study of blood rheological properties in patients with coronary heart disease with laser-optic techniques [1 1065-90]

11065 OV Dielectrophoresis erythrocytes images for predicting stroke recurrence based on analysis of hemorheological parameters [1 1065-96]

11065 OW An algorithm for speckle noise reduction in endoscopic optical coherence tomography structural imaging [1 1065-53]

$110650 \mathrm{X} \quad$ Young's modulus evaluation for blood vessel equivalent phantoms using optical coherence elastography [11065-54]

11065 OY Borderline reconstruction of absorbing and scattering inhomogeneity in biological tissue using time-resolved diffuse optical tomography [1 1065-55]

$11065 \mathrm{OZ} \quad$ Neuroimaging technique using time-resolved diffuse optical tomography and inhomogeneity localization algorithm [1 1065-56]

1106510 Crystallography of oral fluid as an element of personalized evaluation of the human organism's functional state [1 1065-57]

$1106511 \quad$ New approach to detect skin pathologies with polarimetric detection and processing [1 1065-78]

THERAPY AND SURGERY LASER APPLICATIONS

1106512 In vitro Yb,Er:Glass laser hydroacoustic processing of human cataract eye lens: influence of pulse structure on removal efficiency [11065-4]

1106513 Optical and morphological investigation of oral cavity mucosa regeneration after the fractional treatment by radiation of $\mathbf{9 8 0} \mathbf{~ n m}$ diode laser [1 1065-67]

$1106514 \quad$ Optical and thermal modeling of Ti-doped optothermal fiber converter for laser surgery [1 1065-105]

1106515 The peculiarities of localized laser heating of a tissue doped by gold nanostars [11065-104]

1106516 Modeling of hyperthermia induced by functionalized gold nanorods bound to Staphylococcus aureus under NIR laser radiation [1 1065-107]

1106517 Photodynamic therapy of onychomycosis by high-intensive LED light of wavelength $660 \pm 10$ nm [1 1065-16]

1106518 Photodynamic aspects of antimicrobic action of silvernanoparticles on Staphylococcus aureus strains [1 1065-301]

1106519 Clinical studies of the combined action of ultraviolet and laser (662 $\mathbf{n m}$ ) radiation with methylene blue for local therapy of defects of oral mucosa in chronic recurrent aphthous 
stomatitis [1 1065-108]

MICRO- AND NANOBIOTECHNOLOGY

$110651 \mathrm{~A} \quad$ SERS detection of some drugs using aluminum hydroxide with embedded copper nanoparticles [1 1065-6]

11065 1B Investigation of polyelectrolyte microcapsule aggregation in human blood [1 1065-85]

11065 IC Influence of saline background on microstructured optical fibers optical properties [1 1065-73]

11065 1D The pH of protein solutions effect on microstructured optical fibers transmission spectrum [1 1065-80]

11065 IE High-temperature terahertz intrawaveguide spectroscopy using hollow-core sapphire photonic crystal waveguide [1 1065-99]

$110651 \mathrm{~F} \quad$ Electrospark alloying of zirconium and subsequent induction heat treatment [1 1065-46]

$110651 \mathrm{G}$ Analysis of (Ti, $\mathrm{Zr}, \mathrm{Ta})-(\mathrm{O}, \mathrm{C}, \mathrm{N})$ systems for obtaining metal-ceramic coatings of the required composition by induction heat treatment [1 1065-89]

$110651 \mathrm{H}$ The absorption of oxygen and hydrogen by titanium during heat treatment with high frequency currents [1 1065-93]

$1106511 \quad$ Optical and scanning electron microscopy of the metal-oxide inserts [1 1065-94]

$110651 \mathrm{~J} \quad$ Ultrafast all-optical THz modulation based on wavelength scaled dielectric particle with graphene monolayer [1 1065-95]

$110651 \mathrm{~K}$ On the localization of thermal sources existing in a field emission planar nanostructure with a DLC film on a metal edge [1 1065-98]

$11065 \mathrm{lL}$ Thermal optics of ordered arrays of plasmon nanoparticles in context of SERS, cell optoporation, and pathogen destruction [1 1065-106]

\section{MOLECULAR DETECTION AND STUDY}

$110651 \mathrm{M}$ Improvement of creatinine SERS detection using molecularly imprinted silica gel [1 1065-7]

$110651 \mathrm{~N} \quad$ Factors affecting photoluminescence of structures based on citric acid and ethylenediamine [11065-9]

1106510 Spectroscopic analysis supported by chemometric tools for quality control of plant- and animal-based matrices [1 1065-1 1] 
11065 IP The interaction of the europium complex with human serum albumin [1 1065-59]

$110651 Q \quad$ Changes in the spectral characteristics of preparations containing ascorbic acid with the addition of stabilizers [1 1065-60]

11065 IR Induction of chlorophyll fluorescence in Tradescantia leaves acclimated to growth light of different intensities [1 1065-72]

11065 is Study of dielectric parameters of protein solution [1 1065-75]

$110651 \mathrm{~T} \quad$ Optical properties of biomolecular complexes [1 1065-76]

$110651 \mathrm{~N} \quad$ New techniques for measuring zeta-potential of colloidal system [1 1065-79]

$110651 \mathrm{~V} \quad$ Investigation of Ce6 accumulation and distribution in cell cultures of head and neck cancers [1 1065-83]

$110651 \mathrm{~W} \quad$ Functional and morphological changes in the mother-placenta-fetus system during chronic hypoxia (experimental study) [1 1065-86]

11065 1X Red blood cells interaction mediated by dextran macromolecules: in vitro study using diffuse light scattering technique and optical tweezers [1 1065-88]

$110651 Y \quad$ Diffusion of methylene blue in human dentin in the presence of glucose: in vitro study [11065-101]

1106512 A comparison of terahertz optical constants and diffusion coefficients of tissue immersion optical clearing agents [1 1065-97] 
Proc. of SPIE Vol. 11065 1106501-8 Downloaded From: https://www.spiedigitallibrary.org/conference-proceedings-of-spie on 26 Apr 2023
Terms of Use: https://www.spiedigitallibrary.org/terms-of-use 


\section{Authors}

Numbers in the index correspond to the last two digits of the seven-digit citation identifier (CID) article numbering system used in Proceedings of SPIE. The first five digits reflect the volume number. Base 36 numbering is employed for the last two digits and indicates the order of articles within the volume. Numbers start with $00,01,02,03,04,05,06,07,08,09,0 A, 0 B \ldots . .0 Z$, followed by 10-12, 20-2Z, etc.

Aban'shin, Nikolay P., $1 \mathrm{~K}$

Agranovich, llana, 04, OB

Akchurin, Garif G., 15, 16, 1K, 1L

Akchurin, Georgy G., 15, 16, $1 \mathrm{~L}$

Alyanov, Alexandr L., 08

Amouroux, Marine, 05

Antropova, Maria M., 13

Avetisyan, Yuri A., 15, 16, 1K, $1 \mathrm{~L}$

Badaeva, Elena $\mathrm{V}_{\text {., }} 1 \mathrm{~A}$

Bakal, Artem A., $1 \mathrm{~N}$

Bakulev, A. L., OG, $\mathrm{OH}$

Baranov, Maksim, is

Barshtein, Gregory, 1X

Bashkatov, Alexey N., 05, 09, IW

Basko, Elizaveta A., 09

Belikov, Andrey V., 12, 13, 14, 17

Bespalova, N. V., 18

Bliznuks, D., OA

Blondel, Walter, 05

Bolochko, K., OA

Bolotine, Lina, $1 \mathrm{~V}$

Borisova, Ekaterina, $04, \mathrm{OB}$

Borisova, Nataliya E., IP

Bratashov, Daniil N., 1B

Bryanskaya, Svetlana A., OS

Bucharskaya, Alla B., 1W

Burmak, Liudmila, OC

Burmistrova, Natalia A., 1C, 1D, 10

Chekhonatskaya, Marina L., IW

Chen, Hang, 05

Cherkasova, Olga P., 12

Chuchupal, Sergey V., $1 Z$

Danyaeva, Julia, 1Q

Demidov, Valentin, 02

Demidova, Olga, 02

Derjabo, A., OA

Diachuk, Larisa I., OU

Dobdin, S. YU., OL, OM

Dolganova, Irina N., $1 Z$

Dremin, Viktor $\mathrm{V} ., 08,0 Q$, OR, OT

Dunaev, Andrey $\mathrm{V}$., 08, OQ, OR, OS, OT

Dykman, L. A., OF, 00

Egorov, Ivan S., 1G, 11

Ermakov, Alexey V., 1B

Ermolaeva, Luidmila A., 13

Ermolinskiy, Petr B., 07, OU

Farat, Oleg K., 1P

Farrakhova, Dina, 1V

Fedotov, Denis Y., 13
Feng, Wei, 05

Feodorova, V. A., OF, OG, OH, Ol, OJ, OK, OL, OM, $\mathrm{ON}, 0 \mathrm{O}, \mathrm{OP}$

Filina, Mariya A., 08

Filonova, N. N., OF, OG, OH, Ol, OJ, OK, OL, OM, ON, OO, OP

Flueraru, Costel, 02

Fomin, Aleksandr A., 1H, 11

Fomina, Marina, $1 \mathrm{G}, 1 \mathrm{H}$

Frolov, S. V., OW, OX, OY, OZ

Gagarsky, Sergey V., 12

Galitskaya, Anna, OB

Galushka, Viktor, $1 \mathrm{H}$

Gavdush, Arsenii A., 1 Z

Gelfond, Mark L., 17

Gelikonov, Grigory V., 02, 03

Gelikonov, Valentin M., 03

Generalov, Vladimir M., OV

Genin, Vadim D., 05

Genina, Elina A., 05, 09, 1W

Gladkova, Natalia D., 02, 03, 06, 0D

Gorbachev, llya A., 1A

Goryacheva, Irina Yu., 1N

Grashkina, I. G., ON, 00

Gromov, Andrey A., OV

Gubarev, Fedor A., OE

Gubarkova, Ekaterina V., 03, 06, OD

Gurfinkel, Yury I., OU

Gusel'nikova, Valeriia V., 13

Kaliviotis, Efstathios, 07

Kalmatskaya, Olesya A., 1R

Kandurova, Ksenia Y., 08

Kanevsky, Matvey, OB

Kapitonova, Mariya A., $1 \mathrm{P}$

Karashtin, Dmitry A., 02

Karavaev, Vladimir A., 1R

Karmakova, Tatyana, IV

Katyba, Gleb M., 1E

Khairallah, Grégoire, 05

Kharcheva, Anastasiia V., 1P

Khorovodov, Alexander, 04, OB

Kiseleva, Elena B., 06, OD

Klementeva, Maria $\vee ., 09$

Kolosova, A. A., ON, OP

Komandin, Gennady A., $1 Z$

Konnova, Svetlana, OB

Korneeva, Ekaterina, 11

Korzhevsky, Dmitriy E., 13

Koshuro, Vladimir A., 1F, 11 
Kozlov, Igor O., OQ, OR, OS, OT

Kozlova, Anastasiia A., 1B

Krivetc, Olga O., 10

Kruchinin, Vladimir N., OV

Kruchinina, Margarita $\mathrm{V}$., OV

Kurlov, Vladimir N., 1E

Kutsenko, Svetlana, 1Q

Kuzmina, I., OA

Kuznetsov, Sergey S., 06

Kuznetsova, Irina A., OD

Larionova, O. S., OF, OL, OM, OO

Lazareva, Ekaterina N., 05

Lengert, Ekaterina V., 1B

Li, Lin, OE

Lihacova, I., OA

Loktionova, Yulia I., OS

Loschenov, Victor, 1V

Lugovtsov, Andrei E., 07, 0U, 1X

Lukinsone, $\mathrm{V}$., OA

Lyapina, A. M., OF, OG, OH, Ol, OJ, OK, OL, OM, ON, OO, OP

Machikhin, Alexander, OC

Maklygina, Yulia, $1 \mathrm{~V}$

Makovik, Irina N., OT

Mamedova, Aysel, 04, OB

Mamoshin, Andrian $\mathrm{V}$., $08,0 Q$

Margaryants, Nikita B., OT

Markin, Alexey V., 1A, 1M

Markina, Natalia E., 1A, 1M

Martynov, Dmitry V., 1D

Maslyakova, Galina N., IW

Matveev, Lev A., 02, 03, 06, OD

Matveyev, Alexander L., 02, 03, 06, OD

Mezentsev, Mikhail, OQ, OR

Mikerov, A. N., 19

Millard, Marie, 1V

Minin, lgor $V ., 0 V, 1 \mathrm{~J}$

Minin, Oleg $\vee ., O V, 1 \mathrm{~J}$

Modin, Mikhail A., 17

Moiseev, Alexander A., 02

Monakhova, Yulia B., 10

Mordovina, Ekaterina A., $1 \mathrm{~N}$

Mulik, Alexander B., 10

Muradyan, Vadim F., 08

Musina, Guzel R., 1 Z

Navolokin, Nikita, 04, OB

Nechaeva, O. V., 18

Nepomnyashchaya, Elina, $1 \mathrm{~T}$

Novochadov, Valery $\vee ., 10$

Olomskaya, Vera V., iN

Palatova, Tatyana V., IW

Pankratov, Andrey, IV

Papshev, Vyacheslav A., 11

Patsaeva, Svetlana V., $1 P$

Pavlikova, Elena P., OU

Pavlova, Nadezhda P., 03

Pekker, Yakov S., OE

Piavchenko, Gennadii, $0 Q$

Pidenko, Pavel S., 1C, 1D

Plekhanov, Anton A., 03, 06
Plorina, E. V., OA

Plyutinskaya, Anna, 1V

Poeschl, Christina, 07

Polschikova, Olga, OC

Popov, Ivan, 02

Postnova, Margarita $V ., 10$

Potapova, Elena V., 08, OR

Potemkin, Andrey $V .$, OT

Potlov, A. Yu., OW, OX, OY, OZ

Presnyakov, Kirill $Y ., 1 C$

Priezzhev, Alexander $\mathrm{V} ., 07,0 \mathrm{U}, 1 \mathrm{X}$

Proskurin, S. G., OW, OX, OY, OZ

Putintseva, Maria, 11

Rafailov, Edik U., OS

Rakotomanga, Prisca, 05

Rodionov, Igor, 11

Romanishkin, Igor, $1 \mathrm{~V}$

Ryabova, Anastasia, $1 \mathrm{~V}$

Salogubova, Irina $S ., 14$

Saltykov, Yu. V., OF, OG, OH, OI, OJ, OK, OL, OM, ON, OO, OP

Savchenko, Ekaterina, $1 \mathrm{U}$

Sdobnov, Anton, $0 \mathrm{Q}$

Selifonov, A. A., 19, 1Y

Semenov, Alexei N., 07, $1 \mathrm{X}$

Semyachkina-Glushkovskaya, Oxana, 04, OB

Semyashkina, Yulia V., 13, 17

Serdobintsev, Alexey, $1 \mathrm{H}$

Sergeev, Andrey N., 12

Sergeeva, Elena I., 17

Sergeeva, Elena S., 13

Seryogina, Evgenya, $\mathrm{OQ}$

Shabanov, Dmitry V., 03

Shapoval, O. G., 19

Shatyr, Yulia A., 10

Shchelkunov, Andrey, $1 \mathrm{G}$

Shelkunov, Andrey Yu., 11

Shih, Wei-Chuan, $1 \mathrm{~L}$

Shimanova, Veronika, OB

Shin, Sehyun, 1X

Shnitenkova, Anastasia, OB

Shulgina, T. A., 18

Shumilin, Aleksandr, $1 \mathrm{G}$

Shupletsov, Valery, OR

Shuvalov, Andrei A., $1 \mathrm{C}$

Shuvalov, Gennadiy V., OV

Sidorov, Victor $\vee$., OS

Sirotkina, Marina A., 02, 06

Skaptsov, Aleksandr, 1G, 1H

Skripal', An. V., OL, OM

Skrypnik, Alexei V., 14

Smirnov, Sergey N., 12

Smolyanskaya, Olga A., 1 Z

Soboleva, Polina M., 10

Sokolovski, Sergei G., OS

Soussen, Charles, 05

Sovetsky, Alexander A., 02, 03, 06, OD

Spigulis, J., OA

Stavtsev, Dmitry D., OT

Stelmashchuk, Olga, $\mathrm{OQ}$ 
Subbotina, I. A., OF, OG, OH, OI, OJ, OK, OL, OM, ON, $00,0 P$

Sytnik, Iuliia D., OE

Terskov, Andrey, 04

Tikhomirova, E. I., 18

Tikhonov, Alexander N., 1R

Timofeeva, Lydia B., OD

Tsyupka, Daria $V_{\text {., }} 1 \mathrm{~N}$

Tuchin, Valery V., 05, 09, 15, 16, 19, 1L, 1Y, $1 \mathrm{Z}$

Tuchina, Daria K., 12

Tuchina, Elena S., 16, 1L

Ulesikova, Irina $V_{\text {., }} 10$

Ulianova, O. V., OF, OG, OH, Ol, OJ, OK, OL, OM, ON, $00, \mathrm{OP}$

Ulyanov, S. S., OF, OG, OH, OI, OJ, OK, OL, OM, ON, OO, OP

Ushakova, O. V., 18

Vashenko, Tatiana, OB

Veilande, R., OA

Velichko, Elena, 11, $1 \mathrm{U}$

Verkhovskii, Roman A., 1B

Vitkin, I. Alex, 02

Vlasova, Alina, OC

Volkov, Mikhail V., OT

Vorontsov, Alexey Yu., OD

Vorontsov, Dmitry A., OD

Vostrikova, Anna M., 1N

Voyko, Aleksey, $1 \mathrm{G}$

Windberger, Ursula, 07

Yakavets, llya, $1 \mathrm{~V}$

Yakunin, Alexander N., 15, 16, 1K, $1 \mathrm{~L}$

Yuvchenko, S. A., 19

Zagaynova, Elena V., 02, 03, 06

Zagorulko, Alexey M., 12

Zaitsev, Vladimir Yu., 02, 03, 06, OD

Zakharevich, Andrey M., 1A, 1G, 1H, 1I, 1M

Zanishevskaya, Anastasia A., 1D

Zarkov, Sergey V., 15, 16, $1 \mathrm{~L}$

Zaytsev, Kirill I., 1E, 12

Zaytsev, S. S., OF, OG, OH, OI, OJ, OK, OL, OM, ON, $00,0 \mathrm{P}$

Zaytsev, Sergey M., 05

Zharkikh, Elena V., OS

Zherebtsov, Evgeny A., 08, OR, OS, OT

Zherebtsova, Angelina I., OS

Zhu, Dan, 05

Zimnyakov, D. A., 19

Zykova, Lidia, OC 
Proc. of SPIE Vol. 11065 1106501-12

Downloaded From: https://www.spiedigitallibrary.org/conference-proceedings-of-spie on 26 Apr 2023 Terms of Use: https://www.spiedigitallibrary.org/terms-of-use 


\title{
Conference Committee
}

\author{
Symposium Chair
}

Valery V. Tuchin, Saratov State University (Russian Federation) and National Research Tomsk State University, (Russian Federation) and Institute of Precision Mechanics and Control, RAS (Russian Federation)

Conference Chairs

Metin Akay, University of Houston (United States)

Alexey N. Bashkatov, Saratov State University (Russian Federation) and National Research Tomsk State University (Russian Federation)

Ivan V. Fedosov, Saratov State University (Russian Federation)

Elina A. Genina, Saratov State University (Russian Federation) and National Research Tomsk State University (Russian Federation)

Vyacheslav I. Kochubey, Saratov State University (Russian Federation)

Vladimir N. Kurlov, Institute of Solid State Physics of the RAS (Russian Federation)

Kirill Larin, University of Houston (United States)

Alexander B. Pravdin, Saratov State University (Russian Federation)

\section{Conference Program Committee}

Alexey N. Bashkatov, Saratov State University (Russian Federation) and National Research Tomsk State University (Russian Federation)

Walter Blondel, Université de Lorraine (France)

Ekaterina G. Borisova, Institute of Electronics (Bulgaria)

Alexander V. Bykov, University of Oulu (Finland)

Wei Chen, University of Central Oklahoma (United States)

Kishan Dholakia, University of St. Andrews (United Kingdom)

Maria Farsari, IESL-FORTH (Greece)

Paul M. W. French, Imperial College of Science, Technology \& Medicine (United Kingdom)

James G. Fujimoto, Massachusetts Institute of Technology (United States)

Ekaterina I. Galanzha, University of Arkansas for Medical Science (United States)

Elina A. Genina, Saratov State University (Russian Federation) and National Research Tomsk State University (Russian Federation)

Dmitry A. Gorin, Skolkovo Institute of Science and Technology (Russian Federation)

Steven L. Jacques, Tufts School of Engineering (United States)

Valeriy E. Karasik, Bauman Moscow State Technical University (Russian Federation)

Vyacheslav Kalchenko, Weizmann Institute of Science (Israel)

Nikolai G. Khlebtsov, Institute of Biochemistry and Physiology of Plants and Microorganisms, RAS (Russian Federation) 
Sean J. Kirkpatrick, Michigan Technological University (United States)

Yury V. Kistenev, National Research Tomsk State University

(Russian Federation)

Vyacheslav I. Kochubey, Saratov State University (Russian Federation)

Sergey A. Kozlov, ITMO University (Russian Federation)

Jürgen $\mathbf{M}$. Lademann, Charité Universitätsmedizin Berlin (Germany)

Kirill Larin, University of Houston (United States)

Martin Leahy, National University of Ireland, Galway (Ireland) and Royal College of Surgeons in Ireland (Ireland)

Qingming Luo, Hainan University (China)

Francesco S. Pavone, Università degli Studi di Firenze (Italy)

Alexey P. Popov, University of Oulu (Finland)

Juergen Popp, Leibniz-Institut für Photonische Technologien e.V. (Germany)

Alexander V. Priezzhev, M.V. Lomonosov Moscow State University (Russian Federation)

Igor V. Reshetov, I.M. Sechenov First Moscow State Medical University (Russian Federation)

Alexander P. Savitsky, A.N. Bach Institute of Biochemistry, Research Centre of Biotechnology, RAS (Russian Federation)

Alexander M. Sergeev, Institute of Applied Physics, RAS

(Russian Federation)

Valery V. Tuchin, Saratov State University (Russian Federation) and National Research Tomsk State University, (Russian Federation) and Institute of Precision Mechanics and Control, RAS (Russian Federation)

Ilya V. Turchin, Institute of Applied Physics, RAS (Russian Federation)

Elena V. Zagaynova, Privolzhsky Research Medical University (Russian Federation)

Kirill I. Zaytsev, A.M. Prokhorov General Physics Institute of the RAS (Russian Federation) and Bauman Moscow State Technical University (Russian Federation)

Vladimir P. Zharov, University of Arkansas for Medical Science (United States)

Lihong Wang, Caltech (United States)

Ruikang K. Wang, University of Washington (United States)

Dan Zhu, Huazhong University of Science and Technology (China)

\section{Session Chairs}

$1 \quad$ Plenary Session I

Valery V. Tuchin, Saratov State University (Russian Federation) and National Research Tomsk State University, (Russian Federation) and Institute of Precision Mechanics and Control, RAS (Russian Federation)

2 Plenary Session II

Alexander V. Priezzhev, M.V. Lomonosov Moscow State University (Russian Federation) 
Plenary Session III

Wolfgang Becker, Becker \& Hickl GmbH (Germany)

$4 \quad$ Plenary Session IV

Turgut Durduran, ICFO-Instituto de Ciencias Fotònicas (Spain)

5 Plenary Session $\mathrm{V}$

Kirill Larin, University of Houston (United States)

$6 \quad$ Plenary Session VI

Alexander V. Priezzhev, M.V. Lomonosov Moscow State University (Russian Federation)

$7 \quad$ Plenary Session Internet Biophotonics IV

Valery V. Tuchin, Saratov State University (Russian Federation) and National Research Tomsk State University, (Russian Federation) and Institute of Precision Mechanics and Control, RAS (Russian Federation)

8 Plenary Session VIII

Kirill I. Zaytsev, A.M. Prokhorov General Physics Institute of the RAS

(Russian Federation) and Bauman Moscow State Technical University (Russian Federation)

$9 \quad$ Invited Lecture/Oral Session I

Metin Akay, University of Houston (United States)

10 Invited Lecture/Oral Session II

Valery P. Zakharov, Samara University (Russian Federation)

11 Invited Lecture/Oral Session III

Walter Blondel, Université de Lorraine (France)

12 Poster/Internet Session

Ivan V. Fedosov, Saratov State University (Russian Federation)

Anton Dyachenko, Saratov State University (Russian Federation) 
Proc. of SPIE Vol. 11065 1106501-16

Downloaded From: https://www.spiedigitallibrary.org/conference-proceedings-of-spie on 26 Apr 2023 Terms of Use: https://www.spiedigitallibrary.org/terms-of-use 


\title{
Introduction
}

The Sixth International Symposium on Optics and Biophotonics (Saratov Fall Meeting; SFM18) was held in Saratov, Russian Federation, 24-28 September 2018 with over 500 participants from the Russian Federation, the United States, Canada, Europe, Asia, and Pacific Ocean countries. It covered a wide range of modern problems of fundamental and applied optics, laser physics, photonics, and biomedical optics.

This volume includes selected papers of the following Conferences and Workshops organized in the framework of the Symposium:

\author{
Optical Technologies in Biophysics \& Medicine XX \\ Elina A. Genina and Valery V. Tuchin (Chairs) \\ Microscopic and Low-Coherence Methods in Biomedical and Non- \\ Biomedical Applications XI \\ Kirill Larin and Metin Akay (Chairs) \\ Internet Biophotonics XI \\ Alexey N. Bashkatov, Ivan V. Fedosov, and Valery V. Tuchin (Chairs) \\ Biomedical Spectroscopy $\vee$ \\ Vyacheslav I. Kochubey and Alexander B. Pravdin (Chairs) \\ Advanced Materials for Optics and Biophotonics \\ Vladimir N. Kurlov (Chair)
}

\begin{abstract}
The main attention was paid to the discussion of fundamentals and general approaches of description of coherent, low-coherent, polarized, spatially and temporally modulated light interactions with inhomogeneous absorbing media, low-dimensional structures, tissues, and tissue phantoms in a wide spectral range from $x$-ray to terahertz. Optical properties of various tissues measured in vitro, ex vivo, and in vivo as well as optical biopsy techniques were under consideration. Static and dynamic light scattering in tissues, Doppler, photo-acoustic and photothermal laser-tissue interactions, including nanoparticle doped tissues and cells, light induced mechanical stress, photodynamic effects also were considered. On this basis the variety of laser and optical technologies for medical diagnostics, therapy, surgery, and light dosimetry, as well as for spectroscopy of random and ordered media were presented.
\end{abstract}

SFM18 was organized as the morning plenary sessions, afternoon lectures and oral sessions, evening poster presentations and Internet discussion. Plenary lectures delivered by leading experts in urgent fields of optical and laser life sciences were listened by the attendees with a great interest, and discussed by the audience.

Plenary and invited lectures, as well as oral and poster presentations covered a 
wide area of tissue optics, spectroscopy and imaging, controlling of optical properties of tissues, problems of interaction of terahertz radiation with tissues and tissue-like materials, as well as biophysical and photochemical aspects of photoand laser therapy.

In the framework of the Symposium, a competition for the Best Student Poster Award was organized supported by the SPIE FOCUS Program. Some papers from the winners are included in this volume.

The traditional specific feature of Saratov Fall Meetings is the Internet Session and one-day online discussion. In 2018, this Internet session included 3 plenary lectures, 20 invited lectures and 28 reports.

The papers by the participants from the United States, the Russian Federation, Denmark, Germany, Netherland, Ireland, Italy, Finland, Poland, Israel, China, and more, located at the meeting website: http://sfm.eventry.org/symposium2018//BP11/preliminary, were available during the meeting and will be available for a whole year until the next meeting.

It is a great pleasure and privilege for editors to thank all of the authors for their contributions to the Symposium, especially to the plenary, invited, and Internet lecturers for their exciting presentations.

The organizers of SFM18 are grateful to all the sponsoring organizations and programs that efficiently supported this meeting, especially to: Russian Foundation for Basic Research (Russian Federation); the Russian Academy of Sciences (Russian Federation); SPIE; OSA; IEEE - Institute of Electrical and Electronics Engineers; Russian Technology Platforms "The Medicine of the Future" and "Photonics" (Russian Federation); Government of the Russian Federation; European Technology Platform "Photonics21"; EPIC - European Photonics Industry Consortium; LLC SPE "Nanostructed Glass Technology" (Russian Federation); and RME "INJECT" LLC (Russian Federation); Technolnfo Ltd. (Germany); BioVitrum Ltd. (Russian Federation); and LLC SPE OESSP (Russian Federation).

Elina A. Genina Valery V. Tuchin 


\section{Organizers}

Saratov State University (Russian Federation)

Research-Educational Institute of Optics and Biophotonics at Saratov State University (Russian Federation)

International Research-Educational Center of Optical Technologies for Industry and Medicine "Photonics" at Saratov State University (Russian Federation)

Institute of Biochemistry and Physiology of Plants and Microorganisms of the RAS (Russian Federation)

Institute of Precision Mechanics and Control of the RAS (Russian Federation)

Saratov State Medical University named after V.I. Razumovsky

(Russian Federation)

Volga Region Center of New Information Technologies of Saratov State University (Russian Federation)

National Research Tomsk State University (Russian Federation)

ITMO University (Russian Federation)

Bauman Moscow State Technical University (Russian Federation)

Institute of Solid State Physics of the RAS (Russian Federation)

A.M. Prokhorov Institute of General Physics of the RAS (Russian Federation)

Research Center of Biotechnology of the RAS (Russian Federation)

Biochemical Photonics Committee of Chinese Optical Society (China)

SPIE Student Chapters of Saratov State University (Russian Federation), Bauman

Moscow State Technical University (Russian Federation), Institute of Solid State

Physics of the RAS, and Samara University (Russian Federation)

OSA Student Chapters Saratov State University (Russian Federation) and Bauman

Moscow State Technical University (Russian Federation) 
Proc. of SPIE Vol. 11065 1106501-20

Downloaded From: https://www.spiedigitallibrary.org/conference-proceedings-of-spie on 26 Apr 2023 Terms of Use: https://www.spiedigitallibrary.org/terms-of-use 